\title{
Ganji Formulation for Patients with Hepatocellular Carcinoma Who Have Undergone Surgery: A Multicenter, Randomized, Double-Blind, Controlled Trial
}

\author{
Jing-Hao Zhang $\mathbb{D}^{1,2}$ Chao Zheng, ${ }^{1,2}$ Xiao-Jun Zhu $\left(\mathbb{D},{ }^{1,2}\right.$ Xin Zhang, ${ }^{3}$ \\ Zhi-Jun Hou, ${ }^{1}$ Zhen-Hua Zhou, ${ }^{3}$ Yu-Qing Wang, ${ }^{1}$ Kai-Xia Wang, ${ }^{1,2}$ Zhuo Yu, ${ }^{1,2}$ \\ Man Li $\left(\mathbb{D},{ }^{3}\right.$ Yue-Qiu Gao $\mathbb{D}^{1},{ }^{1,2,3}$ and Xue-Hua Sun $\mathbb{D}^{1,2}$ \\ ${ }^{1}$ Department of Hepatopathy, Shuguang Hospital, Affiliated to Shanghai University of Traditional Chinese Medicine, \\ Shanghai 201203, China \\ ${ }^{2}$ Shanghai Traditional Chinese Medicine Clinical Center of Hepatopathy, Affiliated to Shanghai University of Traditional \\ Chinese Medicine, Shanghai 201203, China \\ ${ }^{3}$ Laboratory of Cellular Immunity, Shanghai Key Laboratory of Traditional Chinese Medicine, \\ Affiliated to Shanghai University of Traditional Chinese Medicine, Shanghai 201203, China \\ Correspondence should be addressed to Yue-Qiu Gao; gaoyueqiu@hotmail.com and Xue-Hua Sun; susan_sxh@sina.com
}

Received 25 January 2019; Revised 10 May 2019; Accepted 10 June 2019; Published 19 June 2019

Academic Editor: Jairo Kennup Bastos

Copyright (C) 2019 Jing-Hao Zhang et al. This is an open access article distributed under the Creative Commons Attribution License, which permits unrestricted use, distribution, and reproduction in any medium, provided the original work is properly cited.

Objective. To ascertain the efficacy and safety of Ganji Formulation (GF) for patients with Hepatocellular carcinoma (HCC) who had undergone surgery. Materials and Methods. A total of 262 HCC patients who had undergone liver resection, local ablation, or transcatheter arterial chemoembolization (TACE) were divided randomly into the treatment group and control group. The former was treated with GF and the later with placebo, both for 6 months. The primary endpoint was overall survival (OS). Second endpoints were disease-free survival (DFS) or time to disease progression (TTP). Results. OS of the treatment group was significantly longer than that of the control group $(P<0.05)$. Subgroup analysis showed that, for patients who received TACE, the TTP was significantly longer in the treatment group than in the control group $(\mathrm{P}<0.05)$. However, for patients who underwent liver resection or local ablation, there was no significant difference in DFS between the two groups $(P>0.05)$. Conclusion. GF could improve postoperative cumulative survival and prolong the TTP. This clinical trial number is registered with ChiCTR-IOR-15007349.

\section{Introduction}

Liver cancer is the fifth most common cancer and second most frequent cause of cancer-related death worldwide. Hepatocellular carcinoma (HCC) represents $\sim 90 \%$ of primary liver cancer and constitutes a major global health problem [1]. HCC in China accounts for $55 \%$ of all HCC cases worldwide [2].

There are various treatment approaches for HCC: resection, liver transplantation, local ablative therapies, transcatheter arterial chemoembolization (TACE), particle radiotherapy, and molecularly targeted treatment [3]. Despite these options, recurrence at 5 years after curative therapies is $\sim 70 \%$, with a poor overall prognosis [4]. Chemotherapy and targeted therapy are associated with a low response rate, severe side effects, and expense [5]. Therefore, effective alternative and complementary approaches are required to improve the outcome for HCC patients.

Traditional Chinese medicine (TCM) has been used for centuries and has an important role in prevention of the recurrence and metastasis of cancer, attenuating toxicity, and prolonging the survival of cancer patients after surgery $[6,7]$. The adjuvant therapeutic effects of some TCM herbs has been identified previously $[4,5,8,9]$. Ganji formulation (GF) is used for HCC in TCM. However, evidence-based data regarding the efficacy and safety of GF is lacking. To address this knowledge gap, we carried out a multicenter, randomized, double-blind, placebo-controlled clinical trial to 
determine the therapeutic role of GF for HCC patients who had undergone surgery.

\section{Materials and Methods}

2.1. Ethical Approval of the Study Protocol. The present study was conducted in accordance with the ethical principles of the Declaration of Helsinki and regulation of clinical trials. The study protocol was approved by the Ethics Committee of Shuguang Hospital, Shanghai University of Traditional Chinese Medicine (2015-390-18-01; Shanghai, China). Written informed consent was obtained from all enrolled patients. The trail was registered in Chinese Clinical Trail Registry on 26th October 2015, and the clinical trial number is registered with ChiCTR-IOR-15007349.

2.2. GF. GF is composed of Dangshen (Codonopsis pilosula (Franch.) Nannf.), Baizhu (Atractylodes macrocephala), Baimaogen (Imperata cylindrica Beauv.var. major (Nees) C.E.Hubb.), Muli (Ostreagigastnunb), Biejia (concha testudo graeca), Zelan (Aconitum gymnandrum Maxim.), Chenpi (Citrus reticulata Blanco), Maozhuacao (Ranunculus ternatus Thunb.), Jigucao (Abrus cantoniensis Hance) Baihuasheshecao (Hedyotis diffusa Willd) Baiying, (Solanum lyratum Thunb.), Banzhilian (Scutellaria barbata D. Don), Biliguo (Ficus pumila Linn.), and Tengligen (Actinidia arguta (Sieb. \& Zucc) Planch. ex Miq.).

Concentrated granules of the 14 herbs described above were provided by Jiang Yin Tian Jiang Pharmaceuticals (Jiangsu Sheng, China) and packaged together according to the prescription of GF (lot number: 1508387). Placebo was also in the form of concentrated granules but composed of $10 \%$ GF and pharmaceutical excipient (dextrin). The latter was used in accordance with regulations on the management of pharmaceutical excipients and hygiene standards for use of food additives in China. The placebo provided by Jiang Yin Tian Jiang Pharmaceuticals shared the same package and label as GF. The appearance and taste of placebo was similar to that of herb granules.

2.3. Criteria for Inclusion/Exclusion. Inclusion criteria were patients aged 18-65 years; with a diagnosis of primary liver cancer (Barcelona Clinic Liver Cancer staging B or C); who had liver resection, ablation (radiofrequency ablation (RFA), microwave ablation (MWA), percutaneous ethanol injection (PEI)), or TACE 3 months previously.

Exclusion criteria were patients with metastatic liver cancer; Barcelona Clinic Liver Cancer staging D; severe complications such as refractory ascites, hepatic encephalopathy, liver failure, or portal venous embolism; other forms of liver disease; other severe primary disease or mental disorder. Also, pregnant and lactating women and patients undergoing sorafenib treatment were excluded.

2.4. Grouping. All patients underwent antiviral therapy, hepatoprotective therapy, diuretic treatment, or anti-infective therapy, as indicated. Patients were assigned randomly to receive GF or placebo ( $12 \mathrm{~g}$, p.o., b.d.) for 6 months, respectively, in treatment and control groups.
2.5. Calculation of Sample Size and Randomization. The sample size was calculated according to the 1-year survival of $84.3 \%$ for HCC patients who had undergone surgery reported by Zhu and colleagues [10] and the assumption that the treatment group would obtain a $>10 \%$ increase in survival. An alpha error of 0.05 and a study power of $\sim 80 \%$ (beta $=0.20$ ) were considered, and a drop-out rate of $20 \%$ was assumed. Hence, $>200$ patients would be required for enrollment into our study. A completely random method was employed, and enrolled patients were assigned into two groups with a proportion of 1:1, and the subjects were simply randomly assigned.

2.6. Follow-Up. Patients were asked to visit 3, 6, 9, and 12 months after randomization. The parameters measured were liver function; renal function; serum levels of alphafetoprotein (AFP); complete blood counts; urine; coagulation; Child-Pugh score; hepatitis-B virus (HBV) serology; and $\mathrm{HBV}$ viral load. Also, we undertook ultrasonography, computed tomography (CT), or magnetic resonance imaging (MRI) of the liver and calculated Eastern Cooperative Oncology Group performance status (ECOG-PS).

2.7. Efficacy Endpoints. The primary endpoint was 1-year cumulative overall survival (OS). OS was defined as the time from enrollment to death by any cause. Secondary endpoints were disease-free survival (DFS) and time to disease progression (TTP). DFS was defined as the time from randomization until any subsequent treatment for recurrent HCC diagnosed using abdominal ultrasonography, contrast-enhanced CT, or contrast-enhanced MRI. TTP was defined as the time from randomization until objective tumor progression diagnosed based on image examination and the serum level of AFP.

2.8. Assessment of Safety. During this trial, all patients were advised to reported changes in health and questioned about adverse events (AEs) at each follow-up point. All AEs were documented, and drug-related AEs were assessed by physicians based on physical examination and laboratory tests.

2.9. Statistical Analyses. The analysis was carried out according to the intention-to-treat principle. Continuous variables are the mean \pm SD and were analyzed by the Student's $t$ test. Categorical variables are described by percentage. Comparison of categorical variables was conducted by Pearson's chi-square test or Fisher's exact test. The log rank test was used to compare the Kaplan-Meier survival curve between two groups. Safety analyses were done only if patients had received GF. $P<0.05$ (two-sided) was considered significant. Statistical analyses were done using SPSS v21.0 (IBM, Armonk, NY, USA).

\section{Results}

3.1. Demographic Features of the Study Population. From September 2015 and December 2017, 262 patients were recruited from three hospitals in Shanghai. After exclusion of 43 patients, 217 eligible patients were assigned randomly to the treatment group (107) or control group (112) in a doubleblinded manner. The baseline characteristics of the patients 
TABLE 1: Demographic and baseline characteristics of patients.

\begin{tabular}{|c|c|c|c|}
\hline Characteristic & Treatment group $(n=90)$ & Control group $(n=90)$ & $P$ \\
\hline Age $\left(\right.$ years) ${ }^{\dagger}$ & $56.74 \pm 8.43$ & $55.24 \pm 10.83$ & 0.301 \\
\hline Male (\%) & $78(86.67)$ & $71(78.89)$ & 0.257 \\
\hline 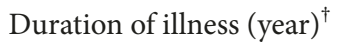 & $3.91 \pm 1.31$ & $3.70 \pm 1.16$ & 0.236 \\
\hline HBsAg positive (\%) & $76(84.44)$ & $71(80.90)$ & 0.060 \\
\hline Child-Pugh score & & & 0.083 \\
\hline A & $48(53.33)$ & $37(41.11)$ & \\
\hline B & $42(46.67)$ & $53(58.89)$ & \\
\hline Total bilirubin $(\mu \mathrm{mol} / \mathrm{L})^{\dagger}$ & $17.51 \pm 11.24$ & $16.07 \pm 8.91$ & 0.387 \\
\hline $\operatorname{Albumin}(\mathrm{g} / \mathrm{L})^{\dagger}$ & $39.96 \pm 4.69$ & $46.35 \pm 44.97$ & 0.161 \\
\hline $\operatorname{ALT}(\mathrm{IU} / \mathrm{L})^{\dagger}$ & $51.08 \pm 42.74$ & $53.01 \pm 37.06$ & 0.683 \\
\hline $\operatorname{AST}(\mathrm{IU} / \mathrm{L})^{\dagger}$ & $47.56 \pm 42.05$ & $52.14 \pm 39.37$ & 0.815 \\
\hline$\alpha$-fetoprotein $(\mathrm{ng} / \mathrm{mL})^{\dagger}$ & $307.82 \pm 257.58$ & $275.23 \pm 141.07$ & 0.484 \\
\hline ECOG score & & & 0.362 \\
\hline 0 & $29(32.22)$ & $27(30.0)$ & \\
\hline 1 & $54(60.0)$ & $60(66.67)$ & \\
\hline 2 & $7(7.78)$ & $3(3.33)$ & \\
\hline Type of treatment & & & 0.357 \\
\hline HR & $22(24.44)$ & $18(20.0)$ & \\
\hline RFA & $7(7.78)$ & $8(8.89)$ & \\
\hline MWA & $6(6.67)$ & $5(5.56)$ & \\
\hline PEI & $4(4.44)$ & $2(2.22)$ & \\
\hline TACE & $51(56.67)$ & $57(63.33)$ & \\
\hline
\end{tabular}

Notes: values in parentheses are percentages; ${ }^{\dagger}$ value is the mean \pm standard deviation.

ALT, alanine aminotransferase; AST, aspartate aminotransferase; ECOG, Eastern Cooperative Oncology Group; HR, hepatic resection; RFA, radiofrequency ablation; MWA, microwave ablation; PEI, percutaneous ethanol injection; TACE, transcatheter arterial chemoembolization.

are shown in Table 1. No significant difference was observed between the groups. A flow diagram of the trial is shown in Figure 1.

3.2. OS. Ten patients in the treatment group and 21 people in the control group died. One-year OS was $88.9 \%$ in the treatment group versus $76.7 \%$ in the control group (chi-square = 4.17, $P=0.030$ ). Kaplan-Meier analysis demonstrated that the treatment group had significantly better cumulative OS compared with the control group (chi-square $=4.431 ; 95 \%$ confidence interval (CI) $0.2262-0.925 ; P=0.0353$ ) (Figure 2).

3.3. DFS and TTP. After 1 year of follow-up, for 40 patients who underwent liver resection and 32 patients who received local ablation (RFA, MWA, and PEI), 53 patients were disease-free (30 in the treatment group and 23 in the control group). No significant difference was observed in DFS between the treatment group and control group $(76.9 \%$ versus $69.7 \%$, chi-square $=0.48 ; P=0.488$ ) (Figure $3(\mathrm{a})$ ). For 108 patients who received TACE, disease progression was observed in 26 of 51 in the treatment group and in 40 of 57 in the control group $(51.0 \%$ versus $70.2 \%$; chi-square = $0.442 ; P=0.041)$. The median TTP in the treatment group and control group was 10 months and 5.2 months, respectively. There was significantly longer TTP in the treatment group compared with the control group (chi-square $=3.983 ; 95 \% \mathrm{CI}$ $=0.352-0.991 ; P=0.046$ ) (Figure 3(b)).
3.4. Safety and AEs. During follow-up, AE prevalence was similar between the two groups (Table 2). Fifty-one of the treatment group and 47 of the control group had AEs, the most being diarrhea, abdominal pain, dyspepsia, and nausea. In addition, abnormal complete blood counts (one or more than one of complete blood counts (CBC) were abnormal) were reported in both groups. All AEs were mild and tolerable.

\section{Discussion}

According to current guidelines for HCC, liver resection, liver transplantation, and local ablation are the most effective treatment methods. For patients with early-stage HCC, liver resection can result in 5-year survival of $60-80 \%$ [5], but with a prevalence of recurrence or metastasis of $40-80 \%$. Also, most new clinically diagnosed cases are of intermediate and advanced stages, and the therapeutic options are limited to TACE, chemotherapeutic agents or radiotherapy, leading to side effects, and disappointing results [11]. Rather than focusing only on the tumor, addressing the overall physiology of the patient is a more effective approach for HCC [12].

The role of TCM as an indispensable part of the diagnosis and treatment of liver cancer has been recognized in various medical guidance documents [13]. TCM has unique advantages for the prevention and treatment of primary liver cancer, especially in the comprehensive treatment of 


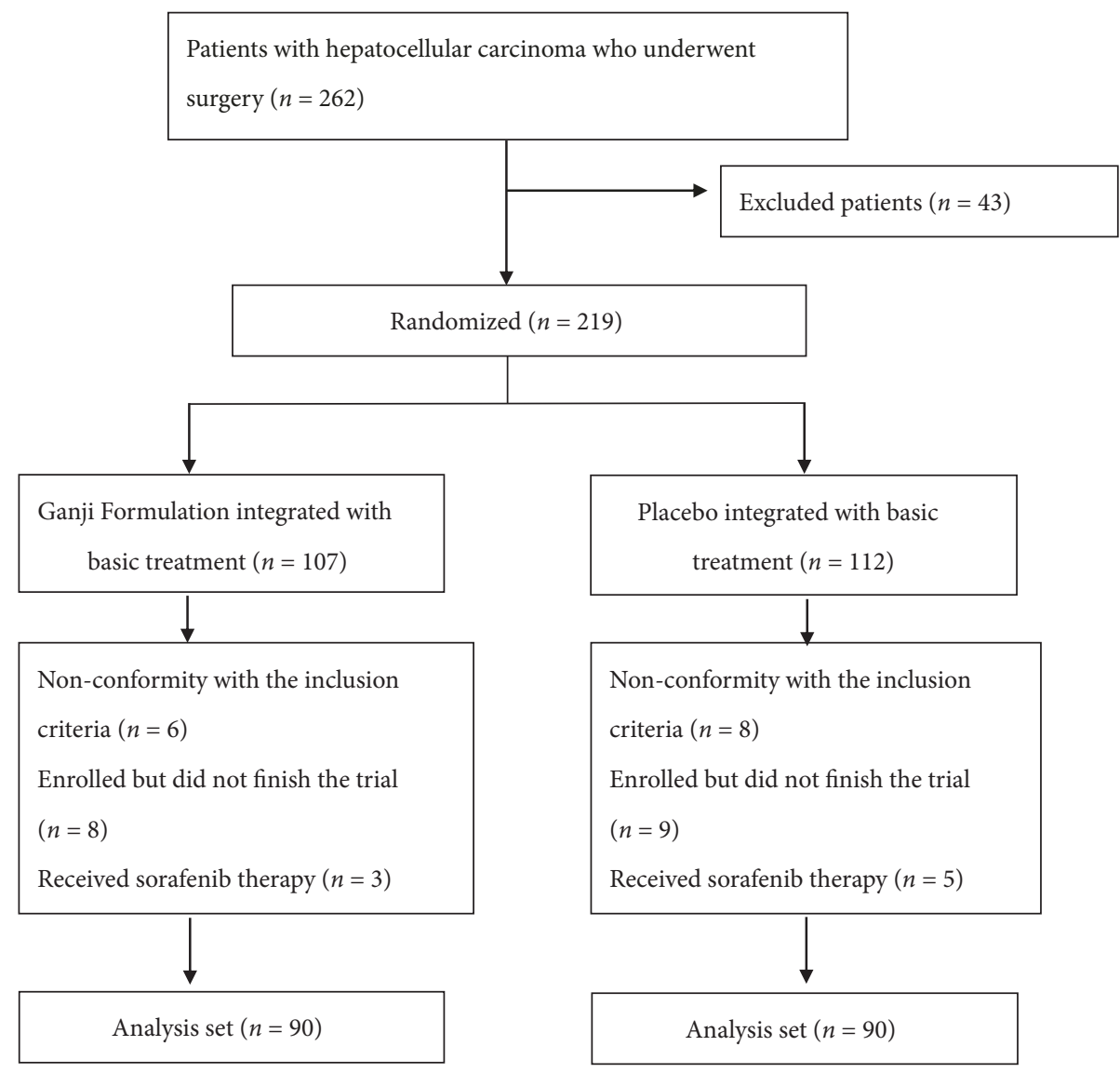

FIGURE 1: Flow diagram of the randomized clinical trial.

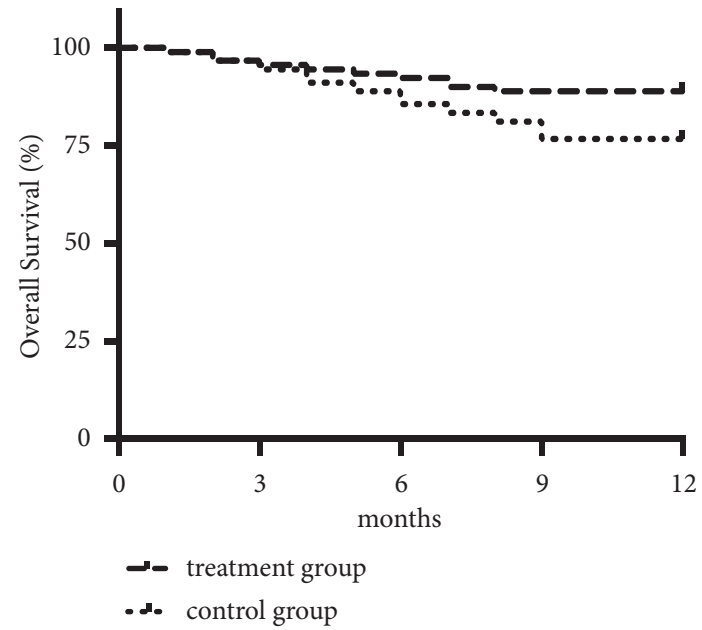

FIGURE 2: Overall survival ratios of treatment group and control group. Significantly better overall survival ratios were observed in treatment group than that in control group $(n=90$ per group, $P=0.0353)$.

advanced HCC. Although there is no direct or local treatment for short-term effects, a combined application can reduce the side effects of surgery, radiotherapy or chemotherapy, improve the symptoms associated with HCC, improve quality of life, and prolong survival. Previously, we showed that the basic TCM syndromes of patients with primary liver cancer who have undergone surgery are spleen deficiency, blood stasis and qi stagnation [14]. Based on previous research, combined with TCM theory for liver tumors and clinical practice, GF was established.

Here, we studied GF as an adjuvant therapy for HCC patients who had undergone surgery. All patients had Barcelona Clinic Liver Cancer staging B or C. The primary endpoint of a significant improvement in OS at 1 year with GF was observed (88.9\% in the treatment group versus $76.7 \%$ in the control group, $P=0.030$ ). With regard to secondary endpoints, DFS between the treatment group and control group did not show a significant difference $(P=0.488)$, and TTP in the treatment group was significantly longer compared with the control group ( $51.0 \%$ versus $70.2 \%, P=0.041)$. Furthermore, we observed that GF could reduce the surface antigen of the HBV (HBsAg) level in HCC patients with chronic HBV infection (data not shown).

GF is a combination of 14 herbal extracts. The active compounds of these herbal extracts can exert antitumor effects in vivo and in vitro. The underlying mechanism includes induction of mitochondria-mediated apoptosis by 2-[(2E)-3,7-dimethyl-2,6-octadienyl]-6-methyl-2,5cyclohexadiene-1,4-dione (MDM) isolated from the aerial part of Atractylodes macrocephala [15] and total flavonoids 
TABLE 2: Adverse events during follow-up.

\begin{tabular}{lccc}
\hline Adverse event & Treatment group $(n=90)$ & Control group $(n=90)$ & $P$ \\
\hline Overall prevalence & $51(56.7)$ & $47(52.2)$ & $2(2.2)$ \\
Fatigue & $4(4.4)$ & $2(2.2)$ & 0.549 \\
Fever & $3(3.3)$ & $5(5.6)$ & 0.678 \\
Diarrhea & $7(7.8)$ & $11(12.2)$ & 0.55 \\
Abdominal pain & $9(10.0)$ & $8(8.9)$ & 0.635 \\
Dyspepsia & $10(11.1)$ & $9(10.0)$ & 0.619 \\
Nausea & $6(6.7)$ & $10(11.1)$ & 0.418 \\
Abnormal CBC & $12(13.3)$ & $0(0)$ & 0.649 \\
Serious adverse event & $0(0)$ & $13(14.4)$ & - \\
Any other complains & $18(20.0)$ & 0.324 \\
\hline
\end{tabular}

Note: values in parentheses are percentages. Abnormal CBC was defined as one or more than one of complete blood counts (CBC) were abnormal. Any other complains include cough, headache, itching, dizziness, arthralgia, etc.

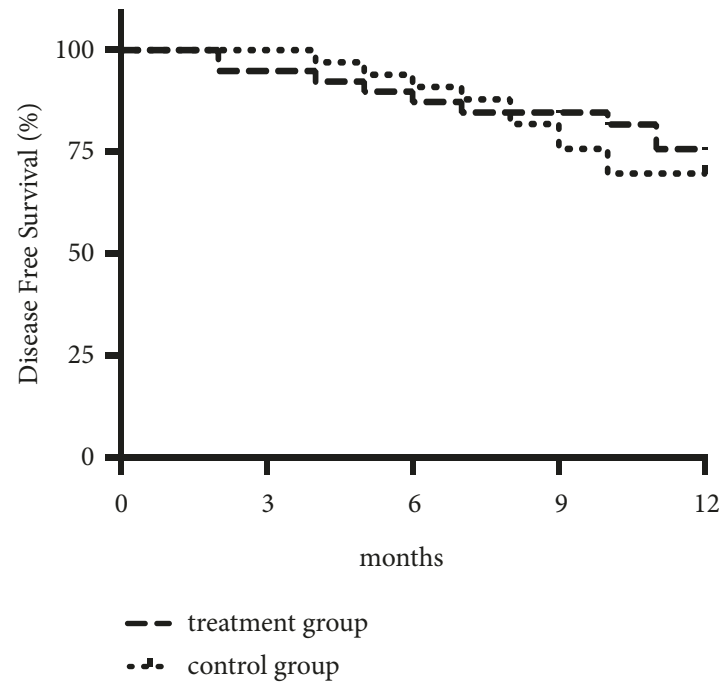

(a)

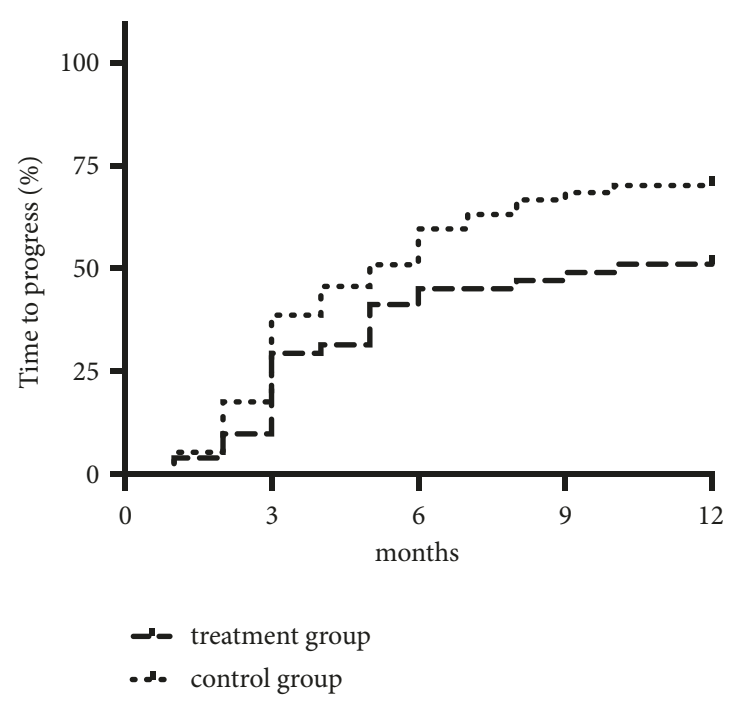

(b)

FIGURE 3: Subgroup analysis of disease-free survival (DFS) and time to progress (TTP). (a) DFS in patients received hepatic resection or local ablation (RFA, MWA, and PEI) between the two groups ( $n=39$ in treatment group and $n=33$ in control group, $P=0.488$ ). (b) TTP in patients received TACE between the two groups ( $n=51$ in treatment group and $n=57$ in control group, $P=0.046$ ).

from Scutellaria barbata D. Don [16], inducing caspasemediated cell death by Abrus agglutinin [17]; inhibition of proliferation of HCC cells by arrest of G2/M and G0/G1 stages of the cell cycle by nobiletin of Citrus reticulata Blanco [18] and total flavonoid glycosides from Actinidia arguta [19] and downregulation of the CDK2-E2F1 pathway by water extract of Hedyotis Diffusa Willd [20]; prevention of the invasion and migration of HCC cells by total saponin from root of Actinidia valvata Dunn [21]; enhancement of the immune system by extract of Solanum lyratum Thunb [22]. In addition, due to the mixture of complex compounds, multiple pathways, and targets of TCM, the effective components and specific mechanisms of actions of GF need to be clarified further.

Our trial had two main limitations. All patients were from Shanghai, and the observation period was only 1 year. Multicenter and long term follow-up clinical studies nationwide need to be carried out to further verify the clinical efficacy of GF for the patients with primary liver cancer.

\section{Conclusions}

We showed that GF combined with basic treatment could prolong OS and delay tumor progression for patients with primary liver cancer who had undergone surgery. Our study shows that GF could be adjuvant therapy for HCC.

\section{Data Availability}

The data used to support the findings of this study are available from the corresponding author upon request.

\section{Conflicts of Interest}

All authors have no conflicts of interest to declare.

\section{Authors' Contributions}

Jing-Hao Zhang and Chao Zheng performed the study and wrote the manuscript. Xin Zhang and Xiao-Jun Zhu analyzed 
the data. Yu-Qing Wang and Zhi-Jun Hou collected and assembled the data. Zhen-Hua Zhou and Zhuo Yu supervised the study and helped in designing the study. Kai-Xia Wang and Man Li proofread the manuscript. Yue-Qiu Gao and XueHua Sun conceived and designed the study. Jing-Hao Zhang and Chao Zheng contributed equally to this work.

\section{Acknowledgments}

This work was supported by National Natural Science Foundation of China (81673935, 81673938, and 81774256), the Three-Year Action Plan for Further Speed Up the Development of Chinese Medicine in Shanghai (ZY(20182020)-CCCX-2003-01; ZY(2018-2020)-CCCX-2003-04), and Shanghai Science and Technology Commission Research Project (16401970600). All authors thank Ling-Xiao Liu of Shanghai Zhongshan Hospital and Xiao-Feng Zhai of Shanghai Changhai Hospital.

\section{References}

[1] J. M. Llovet, M. Ducreux, R. Lencioni et al., "EASL-EORTC clinical practice guidelines: management of hepatocellular carcinoma," Journal of Hepatology, vol. 56, no. 4, pp. 908-943, 2012.

[2] D. Amarapurkar, K. Han, H. L. Chan, and Y. Ueno, "Application of surveillance programs for hepatocellular carcinoma in the asia-pacific region," Journal of Gastroenterology and Hepatology, vol. 24, no. 6, pp. 955-961, 2009.

[3] Y. Takami, S. Eguchi, M. Tateishi et al., "A randomised controlled trial of meloxicam, a Cox-2 inhibitor, to prevent hepatocellular carcinoma recurrence after initial curative treatment," Hepatology International, vol. 10, no. 5, pp. 799-806, 2016.

[4] Q. Chen, C. Shu, A. D. Laurence et al., "Effect of Huaier granule on recurrence after curative resection of HCC: A multicentre, randomised clinical trial," Gut, vol. 67, no. 11, pp. 2006-2016, 2018.

[5] C. Zhong, H.-D. Li, D.-Y. Liu et al., "Clinical study of hepatectomy combined with Jianpi Huayu therapy for hepatocellular carcinoma," Asian Pacific Journal of Cancer Prevention, vol. 15, no. 14, pp. 5951-5957, 2014.

[6] J. Liu, S. Wang, Y. Zhang, H.-T. Fan, and H.-S. Lin, “Traditional Chinese medicine and cancer: History, present situation, and development," Thoracic Cancer, vol. 6, no. 5, pp. 561-569, 2015.

[7] C. Q. Ling, X. Q. Yue, and C. Ling, "Three advantages of using traditional Chinese medicine to prevent and treat tumor," Journal of Integrative Medicine, vol. 12, no. 4, pp. 331-335, 2014.

[8] X.-F. Zhai, Z. Chen, B. Li et al., "Traditional herbal medicine in preventing recurrence after resection of small hepatocellular carcinoma: a multicenter randomized controlled trial," Journal of Chinese Integrative Medicine, vol. 11, no. 2, pp. 90-100, 2013.

[9] Y.-H. Liao, C.-C. Lin, H.-C. Lai, J.-H. Chiang, J.-G. Lin, and T.-C. Li, "Adjunctive traditional Chinese medicine therapy improves survival of liver cancer patients," Liver International, vol. 35, no. 12, pp. 2595-2602, 2015.

[10] Z. Q. Zhu, X. W. Wu, H. Q. Yang et al., "Survival rate analysis and influencing factors of surgical treatment of patients with primary liver cancer," The Journal of Practical Medicine, vol. 29, no. 5, pp. 787-789, 2013.

[11] Z. Yang, X. Liao, Y. Lu et al., "Add-on therapy with traditional chinese medicine improves outcomes and reduces adverse events in hepatocellular carcinoma: a meta-analysis of randomized controlled trials," Evidence-Based Complementary and Alternative Medicine, vol. 2017, Article ID 3428253, 13 pages, 2017.

[12] J. D. Schwartz, M. Schwartz, J. Mandeli, and M. Sung, "Neoadjuvant and adjuvant therapy for resectable hepatocellular carcinoma: Review of the randomised clinical trials," The Lancet Oncology, vol. 3, no. 10, pp. 593-603, 2002.

[13] C. Ling, J. Fan, H. Lin et al., "Clinical practice guidelines for the treatment of primary liver cancer with integrative traditional Chinese and Western medicine," Journal of Integrative Medicine, vol. 16, no. 4, pp. 236-248, 2018.

[14] JH. Zhang, C. Zheng, ZH. Zhou et al., "Research on TCM clinical syndrome distribution of 219 postoperative patients with primary liver cancer," Shanghai Journal of Traditional Chinese Medicine, pp. 51-S1, 2017.

[15] L. Li, R. Zhao, Y. Li, and W.-H. Wang, "Antitumor activity of 2-[(2E)-3,7-dimethyl-2,6-octadienyl]-6-methyl-2,5cyclohexadiene-1,4-dione isolated from the aerial part of Atractylodes macrocephala in hepatocellular carcinoma," Molecular Medicine Reports, vol. 16, no. 5, pp. 6299-6305, 2017.

[16] J. Gao, W.-F. Lu, Z.-J. Dai et al., "Induction of apoptosis by total flavonoids from Scutellaria barbata D. Don in human hepatocarcinoma MHCC97-H cells via the mitochondrial pathway," Tumor Biology, vol. 35, no. 3, pp. 2549-2559, 2014.

[17] S. Mukhopadhyay, P. K. Panda, D. N. Das et al., "Abrus agglutinin suppresses human hepatocellular carcinoma in vitro and in vivo by inducing caspase-mediated cell death," Acta Pharmacologica Sinica, vol. 35, no. 6, pp. 814-824, 2014.

[18] H. Ohnishi, M. Asamoto, K. Tujimura et al., "Inhibition of cell proliferation by nobiletin, a dietary phytochemical, associated with apoptosis and characteristic gene expression, but lack of effect on early rat hepatocarcinogenesis in vivo," Cancer Science, vol. 95, no. 12, pp. 936-942, 2004.

[19] L. Wang, C. Kang, W. Yang et al., "Experimental study on antitumor effects of extracts from Actinidia argutaor," Zhongguo Zhongyao Zazhi, vol. 35, no. 16, pp. 2184-2186, 2010.

[20] X.-Z. Chen, Z.-Y. Cao, T.-S. Chen et al., "Water extract of Hedyotis Diffusa Willd suppresses proliferation of human HepG2 cells and potentiates the anticancer efficacy of low-dose 5-fluorouracil by inhibiting the CDK2-E2F1 pathway," Oncology Reports, vol. 28, no. 2, pp. 742-748, 2012.

[21] G.-Y. Zheng, H.-L. Xin, B. Li, Y.-F. Xu, T.-J. Yi, and C.-Q. Ling, "Total saponin from root of actinidia valvata dunn prevents the metastasis of human hepatocellular carcinoma cells," Chinese Journal of Integrative Medicine, vol. 18, no. 3, pp. 197-202, 2012.

[22] Y. Guan, H. Zhao, X. Yan, J. Meng, and W. Wang, "A study on anti-tumour effect of Solanum lyratum Thunb. extract in $\mathrm{S}_{180}$ tumour-bearing mice," African Journal of Traditional, Complementary and Alternative Medicines, vol. 10, no. 5, pp. 345-351, 2013. 


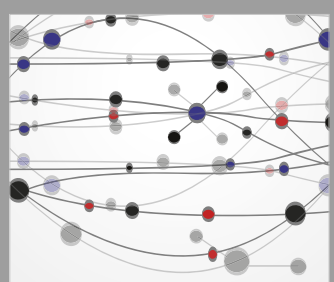

The Scientific World Journal
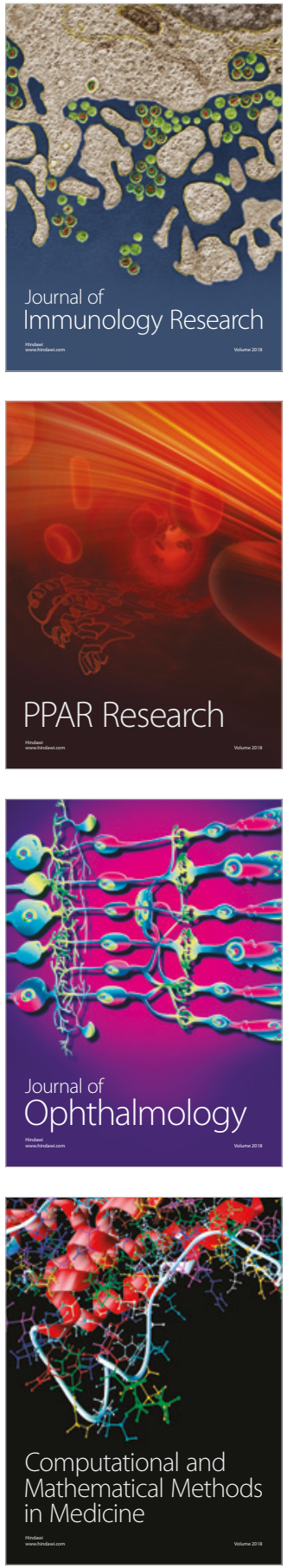

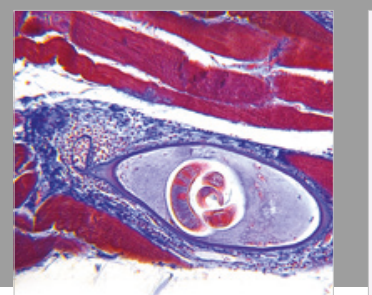

Gastroenterology Research and Practice

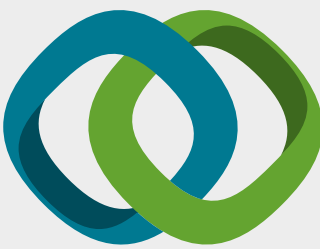

\section{Hindawi}

Submit your manuscripts at

www.hindawi.com
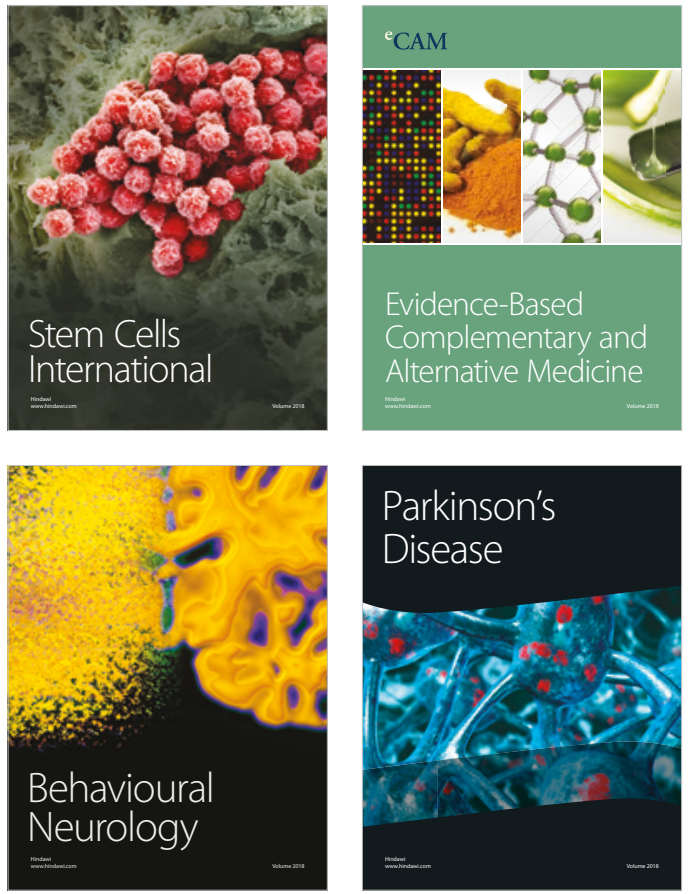

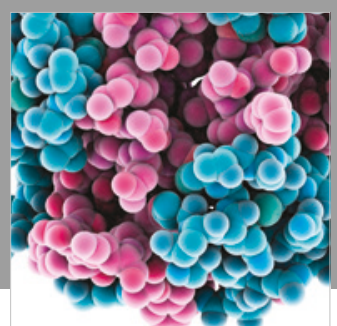

ournal of

Diabetes Research

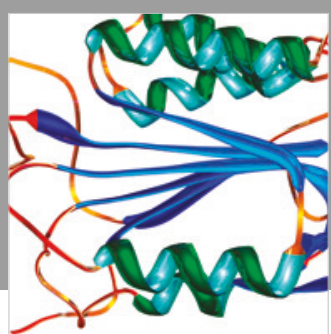

Disease Markers
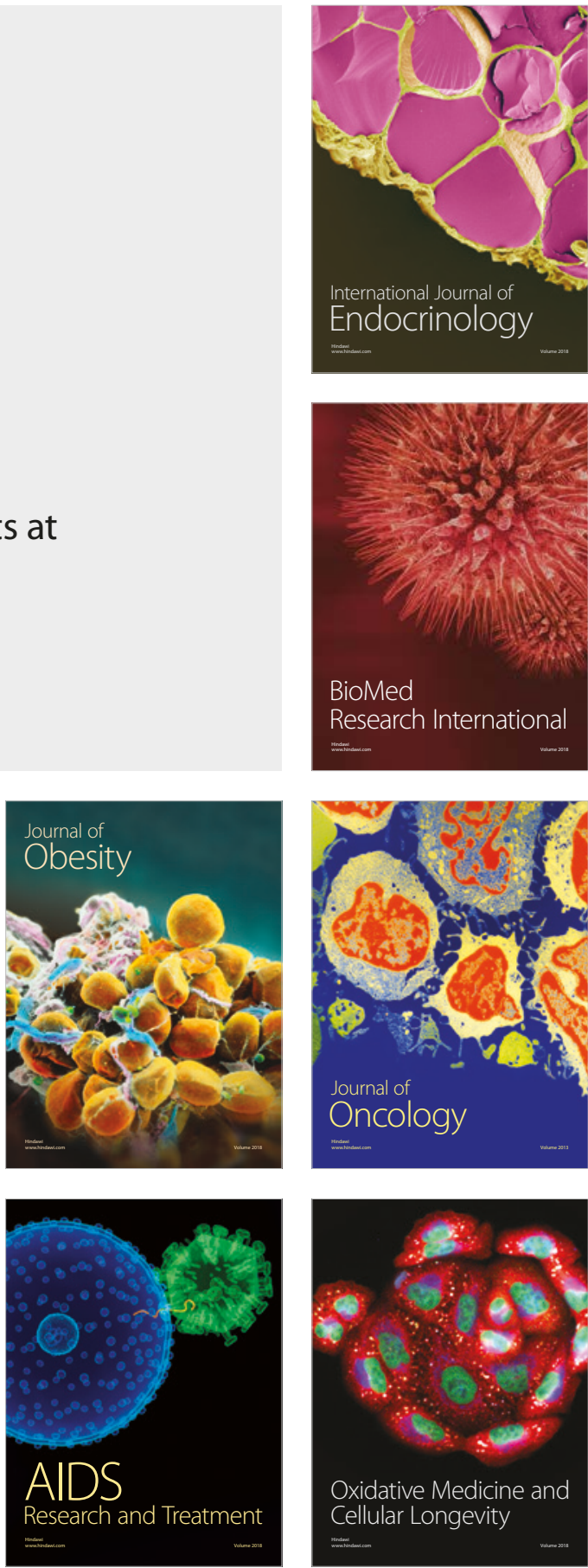